\title{
Erratum to: Improvement of N-glycan site occupancy of therapeutic glycoproteins produced in Pichia pastoris
}

Byung-Kwon Choi • Shannon Warburton • Heping Lin • Rohan Patel • Istvan Boldogh • Michael Meehl •

Marc d'Anjou • Liza Pon • Terrance A. Stadheim •

Natarajan Sethuraman

Published online: 1 June 2012

(C) Springer-Verlag 2012

Erratum to: Appl Microbiol Biotechnol

DOI 10.1007/s00253-012-4067-3

Unfortunately, in the original article, the name of the author Meehl Meehl was given incorrectly.

The correct name is Michael Meehl for which we correctly presented above.

The online version of the original article can be found at http://dx.doi.org/ 10.1007/s00253-012-4067-3.

B.-K. Choi · S. Warburton · H. Lin · R. Patel · M. Meehl •

M. d'Anjou · T. A. Stadheim • N. Sethuraman $(\square)$

GlycoFi, Biologics Discovery, Merck \& Co., Inc,

21 Lafayette St. Suite 200,

Lebanon, NH 03766, USA

e-mail: natarajan_sethuraman@merck.com

I. Boldogh $\cdot$ L. Pon

Department of Pathology and Cell Biology, Columbia University,

630 W. 168th St,

New York, NY 10032, USA 